Europhys. Lett., 13 (8), pp. 679-683 (1990)

\title{
Fluid-Fluid Phase Separation in a Repulsive $\alpha$-exp-6 Mixture: a Comparison with the Full $\alpha$-exp-6 Mixture by Means of Computer Simulations.
}

\author{
A. De KuiJper $\left({ }^{*}\right)$, B. Smit (**), J. A. Schouten $\left(^{*}\right)$ and J. P. J. Michels $\left({ }^{*}\right)$ \\ (*) Van der Waalslaboratorium, Universiteit van Amsterdam \\ Valckenierstraat 67, $1018 \mathrm{XE}$ Amsterdam, The Netherlands \\ (**) Koninklijke/Shell-laboratorium, Amsterdam (Shell Research B.V.) \\ Badhuisweg 3, 1031 CM Amsterdam, The Netherlands
}

(received 13 August 1990; accepted in final form 15 October 1990)

PACS. 05.20 - Statistical mechanics.

PACS. 64.70 - Phase equilibria, phase transitions, and critical points.

PACS. 64.70J - Liquid-liquid transitions.

\begin{abstract}
In this letter we present the results of a study on fluid-fluid phase separation in binary mixtures. We have investigated two systems: a mixture interacting through the full $\alpha$-exp-6 potential and a mixture interacting through the repulsive part of the $\alpha$-exp- 6 potential. Monte Carlo simulations were performed at 300 kelvin and the interaction parameter values used for the full potential correspond to the mixture helium-hydrogen. The results of the repulsive $\alpha$-exp- 6 potential demonstrate clearly that attraction is not necessary for fluid-fluid demixing; its only influence appears to be a shift of the critical pressure. The full $\alpha$-exp- 6 results are in good agreement with the experimental data.
\end{abstract}

The research in (binary-) mixtures is one of the topics which can count on a great deal of interest from both experimental and theoretical physicists nowadays. Not only the wide abundance of mixtures in our everyday life and in our universe but also the surprising new phenomena which were detected in the laboratories are responsible for this increased attention. Mixtures, in general, have a much richer phase diagram than their pure constituents and various effects can be observed only in these multi-component systems. A particular example is the solubility of a fluid in an ordered crystal as was reported recently by Vos and Schouten [1] for the system helium-nitrogen: the dissolved fluid helium causes some of the phase transition lines of pure nitrogen to shift in pressure by at least a factor of two.

One of the most fascinating phenomena in fluid mixtures is the fluid-fluid phase separation. This effect has been studied extensively, in the past and recently, in various binary mixtures both with classical pressure vessel equipment (for pressures up to $10 \mathrm{kbar}$ ) as well as with the diamond anvil cell technique (for pressures up to $100 \mathrm{kbar}$ ). A synopsis of the experimentally studied simple systems consists of $\mathrm{Ne}-\mathrm{Ar}, \mathrm{Ne}-\mathrm{Kr}, \mathrm{Ne}-\mathrm{Xe}, \mathrm{He}-\mathrm{N}_{2}$ and $\mathrm{He}-\mathrm{H}_{2}$. A review of the results for these systems can be found in ref. [2]. 
Computer simulations concerning the fluid-fluid phase behaviour of mixtures have been restricted essentially to hard-sphere systems with the exception of the study on LennardJones systems ( $\mathrm{Ne}-\mathrm{Xe}$ and $\mathrm{Ar}-\mathrm{Kr}$ ) by Panagiotopoulos [3]. In the early sixties [4-6] and more recently $[7,8]$ Monte Carlo simulations and molecular-dynamics calculations have demonstrated that no fluid separation occurs in hard-sphere mixtures (irrespective of the diameter ratio) when additivity is concerned: i.e. $d_{12}=(1 / 2)\left(d_{1}+d_{2}\right)$. Of course, one can always induce demixing by augmenting the unlike interaction parameter $d_{12}$.

In this work we investigate whether fluid-fluid separation is a priori possible in systems which have a purely repulsive interaction only, imposing a physical choice for the interaction parameters. A step forward in answering this question has recently been made by Schouten et al. [9] who have studied the binary inverse-12 system. They concluded on the basis of the simulation data of Hoover et al. [10] that in this system demixing certainly occurs for additive soft spheres when the diameter ratio between the small and large particles is three or larger. Also, using diameter values corresponding to the system helium-hydrogen, qualitative agreement with the experimental data could be obtained. A tendency to homonucleation and thus phase separation had been reported before by Bernu et al. [11] who studied the behaviour of the structure factor $S(k=0)$ through integral equations for a binary inverse-12 mixture with size ratios 1.4 and 1.2. These authors, however, do not refer to a fluid-fluid separation, since this was found well below the glass transition temperature.

Although the inverse-12 system is a much better candidate for the description of the repulsive forces acting between real molecules than the previously studied hard-sphere systems, it cannot be expected to take these forces accurately into account, since it is too steep at short distances and it has an infinite range. In this paper we shall compare a system interacting through the $\alpha$-exp-6 potential with a system interacting through the repulsive part of this potential; i.e. that part of the potential which has $d \phi(r) / d r<0$. The range of this latter potential is restricted to $r<r^{\mathrm{M}}$. We use interaction parameter values corresponding to the mixture helium-hydrogen. The reason for this choice is that the pure components as well as the mixture can be described accurately by the $\alpha$-exp- 6 potential. Evidence for this can be found in ref. [12-14]. Furthermore, $\mathrm{He}^{-\mathrm{H}_{2}}$ has been studied extensively experimentally $[15,16]$ and it is known to exhibit fluid-fluid phase separation up to very high pressures. It is, therefore, the most interesting mixture from the astrophysical point of view. Finally, there have been several theoretical studies on this system amongst which the perturbation theory calculations of van den Bergh and Schouten [14] and the integralequation calculations of Vos et al. [17].

In this study we use the Monte Carlo simulation method of Panagiotopoulos and coworkers [18] which is well suited for our purpose. This method consists of simulating a binary mixture contained in two boxes, keeping the temperature $T$ and pressure $P$ constant. The condition for equilibrium is then the equality of the chemical potentials of each species in the two boxes. Due to the possibility of exchanging particles between the two volumes different compositions may occur, depending on the values of $T$ and $P$. For more technical details on this method we refer to ref. [18] and for a more theoretical description to ref. [19].

At modest pressure we started with an equimolar mixture of 250 particles in each box, randomly placed on a f.c.c. lattice. Then, after each run, the pressure was augmented, using the end configuration of the lower pressure run as starting configuration for the next run. Typically, a run consisted of $1000 \div 2000$ cycles for equilibration and another $1000 \div 2000$ cycles for production. During each cycle all particles in both boxes were attempted to be moved once and the volume of the boxes was varied. We tried to transfer a particle of species $A$ or $B$ (with equal probability) from one box to the other 20000 times; simultaneous swapping of two particles between the boxes was attempted 5000 times. This resulted in several hundreds of particles being transferred and several thousands of particles being 
swapped. Each run was divided into 10 blocks and the estimated uncertainties in the thermodynamical properties correspond to the standard deviations of the block averages. Due to the attempted insertion of particles in both volumes we found, at no additional costs, an estimate for the chemical potentials of both species using the Widom test-particle method [20]. During the run a histogram was constructed on the mole fractions as they occurred in both boxes. Especially when the system is close to the critical point this is important, since both boxes might swap identity during the simulation: e.g., from $A$-rich to $B$-rich. In fig. 1 we show an example of the block averaged mole fractions during the simulation. At the end of a simulation we ran an additional 100 cycles with only particle displacement steps, enabling us to determine the radial distribution functions of the end configuration. These were used to check that the system was in the fluid phase.

We have studied two systems: first a mixture where the particles interact through the full $\alpha$-exp-6 potential,

$$
\phi_{i j}(r)=\frac{\varepsilon_{i j}}{\alpha_{i j}-6}\left\{6 \exp \left[\alpha_{i j}\left(1-\frac{r}{r_{i j}^{M}}\right)\right]-\alpha_{i j}\left(\frac{r_{i j}^{M}}{r}\right)^{6}\right\},
$$

where $i, j \in\left\{\mathrm{He}, \mathrm{H}_{2}\right\}$ and secondly a mixture of particles interacting through the repulsive $\alpha$-exp-6 potential, given by

$$
\phi_{i j}(r)= \begin{cases}\frac{\varepsilon_{i j}}{\alpha_{i j}-6}\left\{6 \exp \left[\alpha_{i j}\left(1-\frac{r}{r_{i j}^{M}}\right)\right]-\alpha_{i j}\left(\frac{r_{i j}^{M}}{r}\right)^{6}\right\}+\varepsilon_{i j}, & \text { if } r \leq r_{i j}^{M}, \\ 0, & \text { if } r>r_{i j}^{M} .\end{cases}
$$

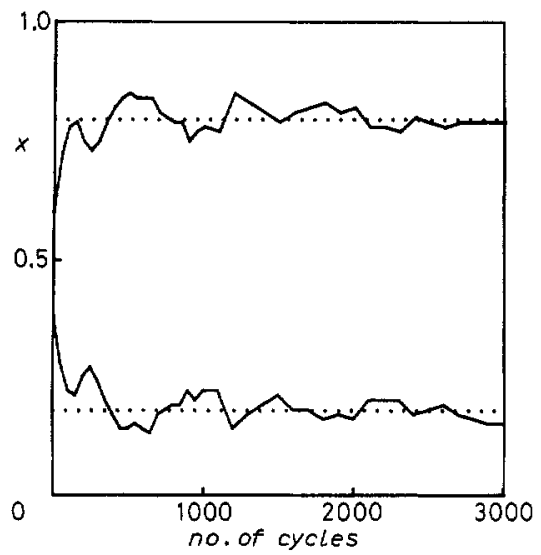

Fig. 1.

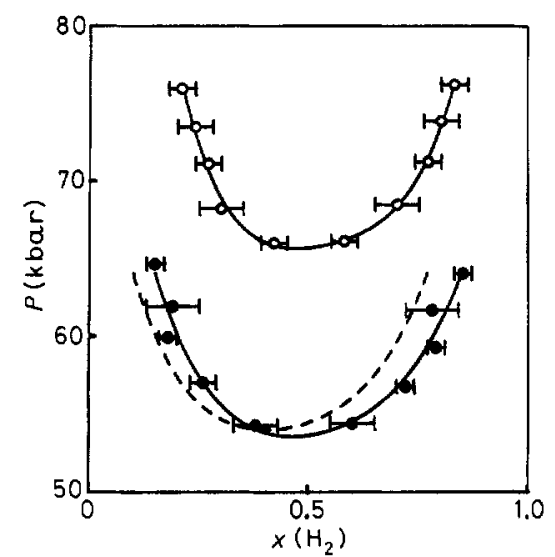

Fig. 2.

Fig. 1. - Block averages of the mole fractions (full lines) in both boxes during 3000 simulation cycles on the $\alpha$-exp- 6 system at $T=300 \mathrm{~K}$ and $P=60 \mathrm{kbar}$ (coming from $\simeq 57 \mathrm{kbar}$ ). The dotted lines indicate the average over the last 1500 cycles.

Fig. 2. - The binodal curves for the $\alpha$-exp- 6 system (filled circles) and the repulsive $\alpha$-exp- 6 system (open circles) at $T=300 \mathrm{~K}$. The dashed line indicates the experimental $\mathrm{He}-\mathrm{H}_{2}$ immiscibility curve from ref. [16]. The full lines serve as guides to the eye. 
In both cases we have used the potential parameter set corresponding to a mixture of helium and hydrogen. The parameter values $\varepsilon_{i j}, \alpha_{i j}$ and $r_{i j}^{\mathrm{M}}$ together with their appropriate references are given in table $\mathrm{I}$. All simulations were performed at $300 \mathrm{~K}$. We did not include quantum contributions (i.e. the Wigner-Kirkwood correction) in our simulations; calculations using perturbation theory performed by us reveal that at $T=300 \mathrm{~K}$, the effect of the quantum corrections on the binodal curve for $\mathrm{He}-\mathrm{H}_{2}$ is a lowering in pressure of about $1.5 \mathrm{kbar}$.

Our main interest is to investigate whether a system interacting through eq. (2) shows fluid-fluid separation and if so, what the differences are with the system interacting through eq. (1). The results for the full $\alpha$-exp-6 system (eq. (1)) are shown in fig. 2 , together with the experimentally determined binodal curve. The simulation result for the critical point is $x_{\mathrm{MC}}^{\mathrm{FUL}}=0.47(2)$ and $P_{\mathrm{MC}}^{\mathrm{FULL}}=53.6(4) \mathrm{kbar}$. This is in good agreement with the experimental data [16]: $x_{\text {EXP }}=0.42$ and $P_{\text {EXP }}=54.0 \mathrm{kbar}$. It should be noted that the unlike interaction parameters come from perturbation theory calculations using the van der Waals one-fluid model; these parameters could certainly be improved. The width of the binodal curve as calculated through the simulations equals the width of the experimentally determined curve. We would like to stress this accordance since this appears to be the most serious shortcoming of the perturbation theory calculations [14]; there the binodal width is highly underestimated.

From fig. 2 it is clear that the potential given in eq. (1) together with the parameter set of table I gives a good description of the helium-hydrogen mixture; eq. (2), using the same

TABLE I. - The parameter values used for the $\alpha-\exp -6$ and the respulsive $\alpha$-exp- 6 potential for the system $\mathrm{He}-\mathrm{H}_{2}$ and their references.

\begin{tabular}{lllll}
\hline Interaction & $\begin{array}{l}\varepsilon / k_{\mathrm{B}} \\
(\mathrm{K})\end{array}$ & $\begin{array}{l}r^{\mathrm{M}} \\
(\AA)\end{array}$ & $\propto$ & Ref. \\
\hline $\mathrm{H}_{2}-\mathrm{H}_{2}$ & 36.4 & 3.43 & 11.1 & {$[12]$} \\
$\mathrm{He}-\mathrm{He}$ & 10.8 & 2.9673 & 13.1 & {$[13]$} \\
$\mathrm{He}-\mathrm{H}_{2}$ & 17.3 & 3.28 & 12.49 & {$[14]$} \\
\hline
\end{tabular}

parameter set, will therefore give a good representation of the repulsive forces present in this mixture. The results for the system without attraction (eq. (2)) are also depicted in fig. 2 (upper curve). From this curve we can draw the important conclusion that, in contrast to liquid-vapour equilibrium, attraction is not needed for fluid-fluid phase separation when we consider «realistic» repulsive interactions. The difference with the fluid-fluid immiscibility curve of the full $\alpha$-exp-6 system is a $12 \mathrm{kbar}$ shift in the critical pressure: $P_{\mathrm{MC}}^{\mathrm{REP}}=65.6(4) \mathrm{vs}$. $P_{\mathrm{MC}}^{\mathrm{FUL}}=53.6(4) \mathrm{kbar}$. The critical composition remains the same: $x_{\mathrm{MC}}^{\mathrm{REP}}=0.47(3)$ vs. $x_{\mathrm{MC}}^{\mathrm{FUL}}=0.47(2)$. Hence, the main influence of attraction appears to be a pressure shift of the binodal curve.

Parts of the necessary computer programs were developed at the Koninklijke/Shelllaboratorium (Shell Research B.V.) in Amsterdam; AdK wishes to thank R. REIJNHART for the warm hospitality during his frequent visits. 


\section{REFERENCES}

[1] Vos W. L. and Schouten J. A., Phys. Rev. Lett., 64 (1990) 898.

[2] Schouten J. A., Phys. Rep., 172 (1989) 33.

[3] Panagiotopoulos A. Z., Int. J. Thermophys., 10 (1989) 447.

[4] Smith E. B. and LeA K. R., Trans. Faraday Soc., 59 (1963) 1535.

[5] Alder B. J., J. Chem. Phys., 40 (1964) 2724.

[6] Rotenberg A., J. Chem. Phys., 43 (1965) 4377.

[7] Fries P. H. and Hansen J.-P., Mol. Phys., 48 (1983) 891.

[8] Jackson G., Rowlinson J. S. and van Swol F., J. Phys. Chem., 91 (1987) 4907.

[9] Schouten J. A., SUN T. F. and DE KuIJPER A., to appear in Physica.

[10] Hoover W. G., Gray S. G. and Johnson K. W., J. Chem. Phys., 55 (1971) 1228.

[11] Bernu B., Hansen J.-P., Hiwatari Y. and Pastore G., Phys. Rev. A, 36 (1987) 4891.

[12] Ross M., Ree F. H. and Young D. A., J. Chem. Phys., 79 (1983) 1487.

[13] Young D. A., McMahan A. K. and Ross M., Phys. Rev. B, 24 (1981) 5119.

[14] vaN DEN Bergh L. C. and Schouten J. A., J. Chem. Phys., 89 (1988) 2336.

[15] Loubeyre P., LeToullec R. and Pinceaux J.-P., Phys. Rev. B, 32 (1985) 7611; 36 (1987) 3732.

[16] Schouten J. A., van den Bergh L. C. and Trappeniers N. J., Chem. Phys. Lett., 114 (1985) 40; van Den Bergh L. C., Schouten J. A. and Trappeniers N. J., Physica A, 132 (1987) 549; Schouten J. A. and VAN DEN BERGH L. C., Fluid phase equilibria, 32 (1986) 1.

[17] Vos W. L., De KuiJPer A., Barrat J.-L. and Schouten J. A., to be published.

[18] Panagiotopoulos A. Z., Mol. Phys., 61 (1987) 813; Panagiotopoulos A. Z., Quirke N., Stapleton M. R. and Tildesley D. J., Mol. Phys., 63 (1988) 527; Stapleton M. R. and Panagiotopoulos A. Z., J. Chem. Phys., 92 (1990) 1285.

[19] SMit B., DE SMedt Ph. and Frenkel D., Mol. Phys., 68 (1989) 931.

[20] SMit B, and Frenkel D., Mol. Phys., 68 (1989) 951. 